\title{
The Jeddah tool
}

\section{$A$ health risk assessment framework for mass gatherings}

\author{
Saber Yezli, BSc PhD, Anas A. Khan, MBBS, SBEM.
}

$\mathrm{M}$ ass gatherings are occasions attended by enough people to strain the preparation and response resources of the host. ${ }^{1}$ They can be spontaneous or planned in advance and sporadic or recurrent in the same site or in different locations. Mass gatherings vary in their nature and may include religious, cultural, sporting, social or political events. ${ }^{1}$ Examples include the FIFA World Cup, music concerts, political rallies, and the Hajj. Mass gatherings can introduce or enhance public health threats, both natural and man made. Risk assessment for mass gatherings enable mass gatherings planners and the host authorities to identify, evaluate, and prioritize these threats so that management and mitigation measures can be implemented. Risk assessment is therefore an integral part of mass gatherings planning and management and a contributor to the success of these events and their long term legacy. ${ }^{1}$

Internationally developed risk assessment standards such as the ISO/DIS31000 provide a generic framework for risk assessment and management. However, there are no recognized mass gatherings-specific risk assessment tools. Health Risk Assessment (HRA) tools developed and implemented in mass gatherings vary in their methodology and scope and are often event-specific and do not always use an all-hazard approach. ${ }^{2,3}$ With the growing frequency, magnitude, and nature of mass gathering events worldwide, there is renewed interest in the development of standardized HRA tools specifically for mass gatherings. ${ }^{3}$

Guided by the United Nations' Sendai Framework for Disaster Risk Reduction, the "Jeddah Tool" designed for HRA of mass gatherings was first developed in 2016 by the Global Centre for Mass Gatherings Medicine, Ministry of Health, Saudi Arabia, to conduct the first strategic HRA for the Hajj. The latter is one of the largest and geographically and culturally diverse annual mass gatherings in the world. The tool was then amended through an international technical consultancy meeting in early 2017 in Jeddah, Saudi Arabia, to produce the updated version of the "Jeddah Tool". ${ }^{4}$ The consultancy meeting included representatives from the World Health Organization (WHO), other WHO collaborating centers on mass gatherings as well as national and international experts from academia and governmental and non-governmental agencies. The "Jeddah Tool" was then used for all-hazard strategic HRAs for successive Hajj seasons (2017, 2018, and 2019). In addition, the tool was adapted for and implemented in other mass gathering events, including the Camel Festival in Saudi Arabia. $^{5}$

The "Jeddah Tool" offers a HRA framework that uses both quantitative and qualitative methods to identify, analyze, evaluate and rank risks associated with mass gathering events and provide recommendations for risk management and mitigation measures. In addition to hazard and vulnerability assessments, the tool also includes a capacity assessment component. The latter evaluates the planning and response capabilities of the host, including the readiness of the health system, as well as identifying capacity gaps, capacity strengthening measures, and optimal distribution of health resources. The capacity assessment component of the "Jeddah Tool" makes the tool particularly useful for recurrent mass gatherings, especially those hosted in the same

From The Global Centre for Mass Gatherings Medicine, Ministry of Health and from the Department of Emergency Medicine, College of Medicine, King Saud University, Riyadh, Kingdom of Saudi Arabia.

Address correspondence and reprint request to: Dr. Saber Yezli, The Global Centre for Mass Gatherings Medicine, Ministry of Health, Riyadh, Kingdom of Saudi Arabia.E-mail: saber.yezli@gmail.com

ORCID ID: https://orcid.org/0000-0002-3380-7092 
location, as it allows for tracking of improvements over events.

A unique feature of the "Jeddah Tool" is that in addition to hazards, vulnerability, and capacity assessments, it also assesses a fourth and significant variable in the context of mass gatherings: reputational risk. Mass gatherings are generally high visibility events, many with political interest and global health security implications. Adverse incidents occurring at a mass gathering can have significant negative ramifications on the reputation of the event itself, its organizers, and the hosting nation or community. This can also lead to damaging political and economic repercussion. The significance of reputational risk in the assessment process may differ depending on the event. For example, the reputational impact related to the Hajj or the Olympic Games may be very different from that of a one-time music festival. The "Jeddah Tool" specifically assesses reputational risk for mass gatherings in a standardized manner. The outcomes of such assessment may potentially facilitate the implementations of the recommended risk management measures by drawing political attention to the findings of the risk assessment.

In summary, the "Jeddah Tool" provides a practical and standardized guide for strategic all-hazard HRA for mass gatherings. The tool extends beyond hazard impact and population vulnerability assessments in detecting

Disclosure. Authors have no conflict of interests, and the work was not supported or funded by any drug company. health system capacity gaps and the reputational consequences of hazards, as well as providing a platform for benchmarking and monitoring health system interventions for recurrent mass gatherings. Given the growing global interest in the mass gatherings health discipline and the increasing number of mass gatherings worldwide, there is an ever-growing need for a standardized HRA tool for these events. The "Jeddah Tool" offers a useful, uniform, and adaptable framework for HRA and management of mass gatherings.

Acknowledgment. We would like to acknowledge Kingsley L. Bieh, Amnah H. Awam, Ahmed S. Elganainy for their valuable contribution to this manuscript.

\section{References}

1. World Health Organization. Public health for mass gatherings: key considerations. Geneva (SW): WHO; 2015.

2. van Dijk A, Dawson E, Moore KM, Belanger P. Risk Assessment During the Pan American and Parapan American Games, Toronto, 2015. Public Health Rep 2017; 132 (1 Suppl): 106S-110S

3. Sharma U, Desikachari BR, Sarma S. Protocol for development of a risk assessment tool for planning and management of religious mass-gathering events of India-a health systemstrengthening initiative. Pilot Feasibility Stud 2019; 5: 83.

4. The Global Centre for Mass Gatherings Medicine. Health Risk Assessment Framework for Mass Gatherings. Jeddah Tool. Available from URL: https://www.moh.gov.sa/Documents/ JED-Tool-v.1-18-7-19.pdf

5. Bieh KL, ElGanainy A, Yezli S, Malik M, Jokhdar HA, Asiri A, et al. Health risk assessment at mass gatherings: a report of the camel festival in Saudi Arabia. East Mediterr Health J 2019; 25: 647-655. 